\title{
Tunable Natural Nano-Arrays: Controlling Surface Properties and Light Reflectance
}

\author{
Jolanta A. Watson ${ }^{\mathrm{a}}$, Sverre Myhra ${ }^{\mathrm{b}}$ and Gregory S. Watson ${ }^{\mathrm{a}}$ \\ ${ }^{a}$ Nanoscale Science and Technology Centre, School of Science, Griffith University; Nathan, QLD, \\ 4111, Australia \\ ${ }^{b}$ Department of Materials Science, University of Oxford, Begbroke Science Park, Sandy Lane, Yarnton, \\ OX5 1PF, UK
}

\begin{abstract}
The general principles of optical design based on the theories of reflection, refraction and diffraction have been rigorously developed and optimized over the last three centuries. Of increasing importance has been the ability to predict and devise new optical technologies designed for specific functions. A key design feature of many of today's optical materials is the control of reflection and light transmittance through the medium.

A sudden transition or impedance mismatch from one optical medium to another can result in unwanted reflections from the surface plane. Modification of a surface by creation of a gradual change in refractive index over a significant portion of a wavelength range will result in a reduction in reflection. An alternative surface modification to the multi layered stack coating (gradient index coating) is to produce a surface with structures having a period and height shorter than the light wavelength. These structures act like a pseudo-gradient index coating and can be described by the effective medium theory. Bernhard and Miller some forty years ago were the first to observe such structures found on the surface of insects. These were found in the form of hexagonally close packed nanometre sized protrusions on the corneal surface of certain moths.
\end{abstract}

In this study we report on similar structures which we have found on certain species of cicada wings demonstrating that the reflective/transmission properties of these natural nano-structures can be tuned by controlled removal of the structure height using Atomic Force Microscopy (AFM).

Keywords: Cicada, Antireflective, Manipulation, Nanostructures, Atomic force microscopy.

\section{INTRODUCTION}

Evolution is a driver of survival technologies, and the survival of the fittest ensures that the most successful technological developments are passed on to successive generations, while those that are less successful are devoured and bred out of existence. Moreover, technologies that arise through evolution must meet a set of holistic criteria, in the sense of being consistent with the great complexity of interactions within the ecosphere. Evolution within the domain of insects must of necessity lead to technologies that are based on nano-scale structures and processes. Thus it can be argued that the insect world offers potential riches to an emerging nano-technology industry, for the same reasons that natural compounds have been a rich source of inspiration and wealth for the pharmaceutical industry.

Naturally occurring nano-structured arrays have been investigated with great interest by the scientific community in order to provide ideal models or templates for man-made fabricated materials and devices. Some forty years ago Bernhard and Miller ${ }^{1}$ made a very interesting discovery whereby certain insects were found to contain closely packed hexagonally ordered nano-metre sized protuberances in their corneal surfaces. The suggestion was made that the function of these arrays was as an anti-reflective medium to light over a large wavelength, thus increasing light transmittance: stealth technology as a survival mechanism against predators? Similar structures have also been found by Yoshida et al. ${ }^{2}$ on the wings of moths (e.g. Cephonodes hylas) Thin film coatings are widely used on a number of different surfaces (e.g., solar cells, lenses, display screens etc.) to suppress undesired reflections and/or increase light transmittance ${ }^{3}$. An 
alternative surface modification to the multi layered stack coating (gradient index coating) is to produce a surface with structures having a period and height shorter than the light wavelength. These structures act like a pseudo-gradient index coating and can be described by the effective medium theory ${ }^{4}$.

In this study we report on nano-structures (a natural pseudo-gradient index coating) which we have found on certain species of cicada wings demonstrating their reflective effectiveness using manipulative atomic force microscopy.

\section{EXPERIMENTAL DETAILS}

\subsection{Materials preparation}

Cicada wings were surgically separated by scalpel. The forewings were cut into smaller sections $(3 \mathrm{~mm} \times 5 \mathrm{~mm})$ and attached via adhesive tape or by glueing directly using an epoxy resin to AFM mounted stubs.

\subsection{Reflectance measurements}

Reflectance measurements were acquired with a THALES OPTEM 100C Series 10:1 Zoom Optical System fitted with a Carl Zeiss 40X/0.60 objective and $10 \mathrm{~mm}$ fibre optic adapter. Light transmission was from 400 to $700 \mathrm{~nm}$. Reflectance data was collected using EPIX XCAP V2.1 software.

\subsection{AFM instrumentation}

The investigations were carried out on two multi-technique/multi-mode instruments; a ThermoMicroscope TMX-2000 Explorer/Discoverer and a JEOL JSPM-4200. The two instruments have broadly comparable capabilities and are both based on detection of tip-to-surface forces through the monitoring of the optical deflection of a laser beam incident on a force-sensing/imposing lever. Several scanners were used in order to attain appropriate image sizes; the fields-of-view ranged from $100 \times 100$ down to $1 \times 1 \mu \mathrm{m}^{2}$. The analyses were carried out under air-ambient conditions (temperature of 20$25^{\circ} \mathrm{C}$ and $40-65 \%$ relative humidity).

\subsection{Electron microscopy}

For scanning electron microscope images a square of dried wing tissue (approx. $3 \mathrm{~mm} \times 5 \mathrm{~mm}$ ) was excised and mounted on an aluminium pin-type stub with double-sided adhesive then sputter coated with 7-10 nm of platinum and imaged using a JEOL $6300 \mathrm{FE}$ scanning electron microscope at $8 \mathrm{kV}$. For transverse sections through wing tissue millimetre wide strips of dried wing tissue were embedded in epoxy resin, polymerised at $60^{\circ} \mathrm{C}$ for 1 day then sectioned with a diamond knife. Sections $70 \mathrm{~nm}$ in thickness were mounted on pioloform (polyvinyl butyral) coated copper grids and stained with lead citrate and uranyl acetate before imaging using a JEOL 1010 transmission electron microscope at $80 \mathrm{kV}$.

\section{RESULTS AND DISCUSSION}

\subsection{Nanostructure Topography}

Two images of the surface of a moth's eye are shown at different lateral resolutions in figure 1. The lower-resolution image in (a) shows the structural surface units of the eye (ommatidial structures). Figure 1 (b) is a higher resolution image which shows the presence of the corneal nipple arrangement consisting of cone-shaped structures displaying the hexagonally close-packed arrangement. The nipple structure shows a spacing of ca. $200 \mathrm{~nm}$. It can be said that the corneal surface possesses a thin, homogeneously transparent layer. As the ratio of cuticle to air changes along the vertical axis which is horizontal to the corneal surface, the refractive index is 1.0 at the top of the array (i.e., refractive index of air), and 1.5-1.6 (chitin material) at the base of the cuticle where the filled volume-fraction is $100 \%$. Thus, as one moves from the top to the bottom of each protuberance, each gradual change in the surface to air ratio will serve as a unit of an antireflective coating with a smooth transitional change in refractive index from 1.0 to 1.5. The mechanism describing the impedance matching between cuticular substrate structure and air has been described by Bernhard and Miller ${ }^{5,6}$. 


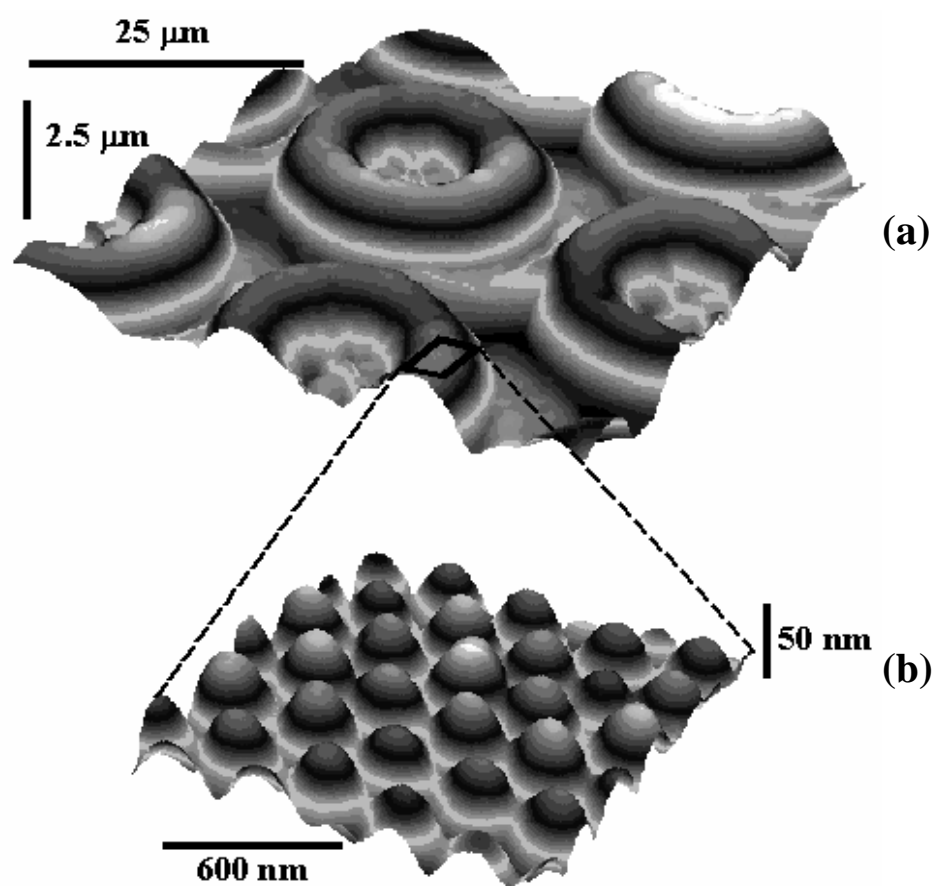

Figure 1. A 3-D image showing the ommatidia structures of a moth eye (a) and a higher resolution image (b) reveals the ordered corneal nipple array.

A grey scale image and line profile shown in figure 2 (a) and (b), respectively, reveals the corneal nipple array of a moth belonging to the family Noctuidae (Speiredonia spectans). The gross change in grey-scale in (a) is the result of the curvature of the ommatidial region. The image also demonstrates the long range hexagonal close-packed order. The line profile in (b) demonstrates the regular periodicity of the structures.

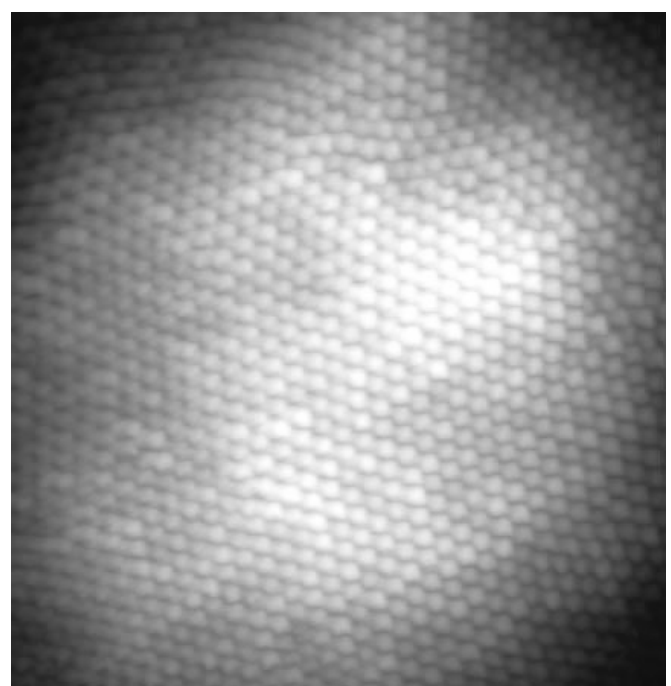

(a)

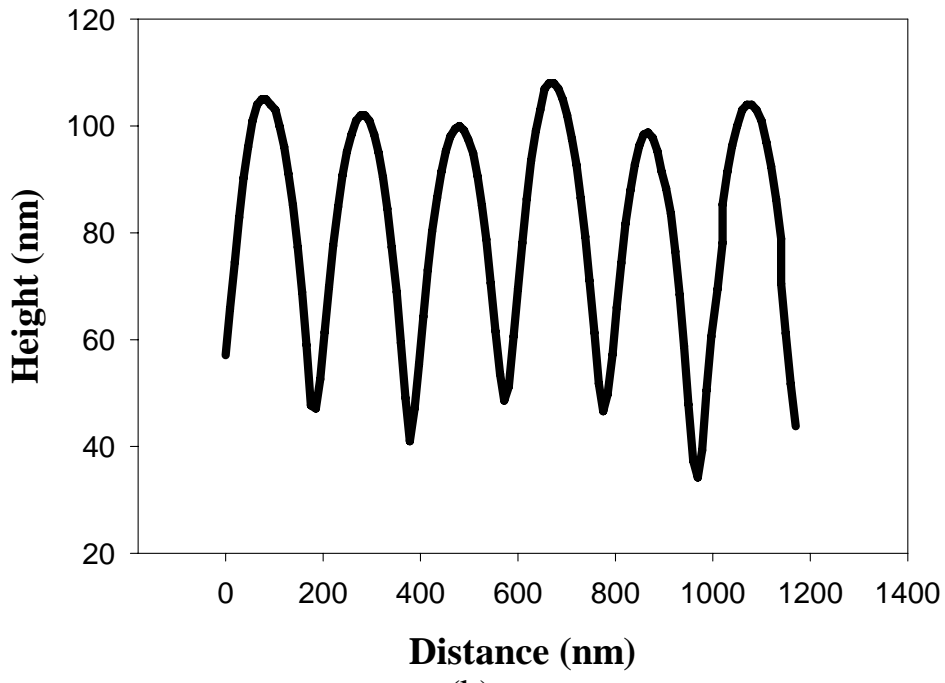

(b)

Figure 2. Topographical grey-scale image (a) and line profile (b) of a moth eye clearly showing the corneal nipple structure.

Similar features to the moth eye arrays have been found on the wings of a number of cicada species (Psaltoda claripennis, Tamasa tristigma, and Cicadetta oldfieldi) (Watson and Watson, 2004) and also Macrotristria angularis and Abricta curvicosta. The nipple arrays were seen on all areas of the dorsal and ventral wing membrane sections. An SEM image of the hexagonally close-packed nanometre sized protrusions on the wing of cicada is shown in figure 3 (a). The 3 dimensional image and line profile (figure 3 (A) and (B), respectively) show the structural details of the cicada array. Detailed structural information can readily be obtained by AFM imaging if the scanning is carried out using a tip with a 
high aspect ratio and small radius of curvature. The line profile shows that the contours of the array from the top to the base of the structures ca be easily traced, thus showing the true profile in three dimensions.

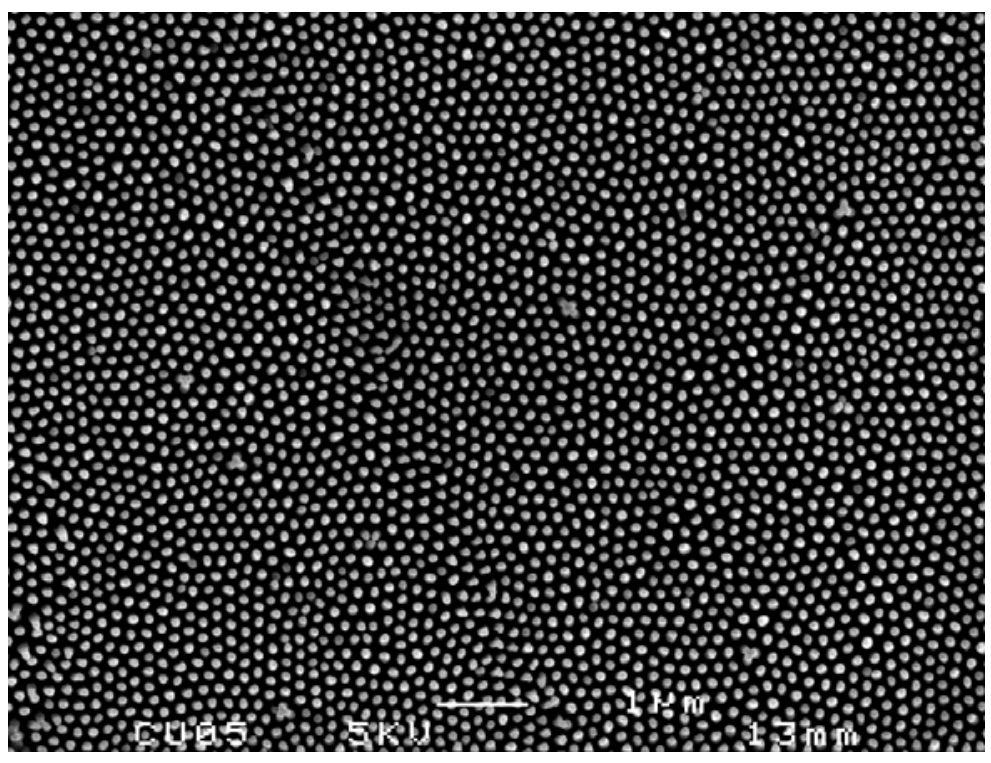

(a)
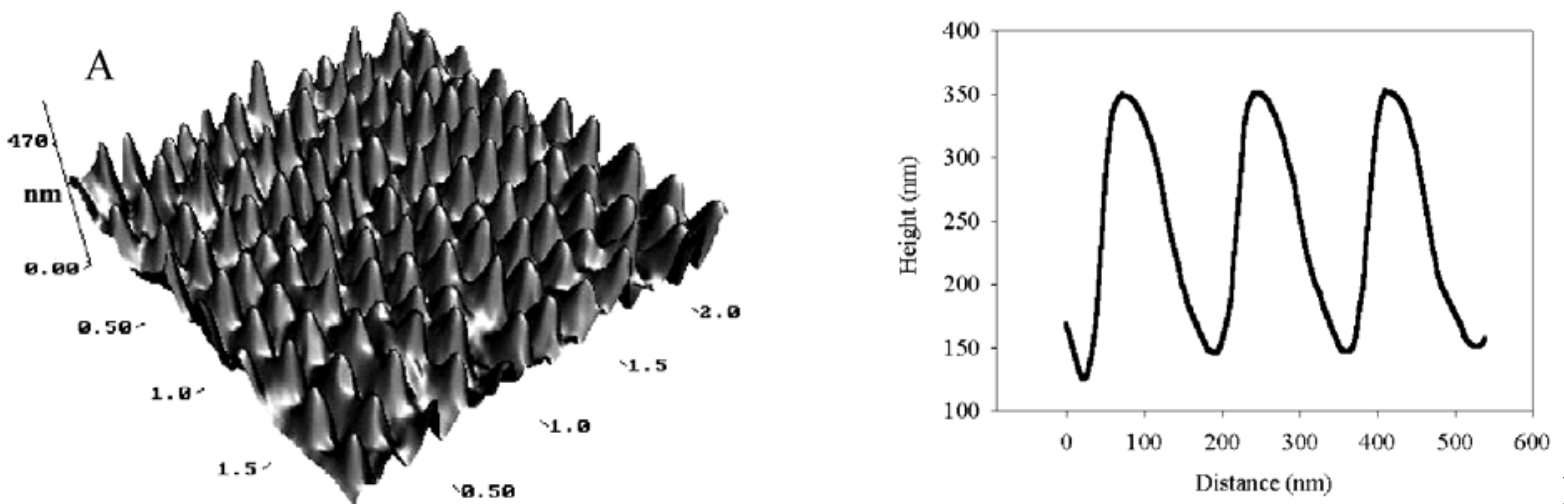

Figure 3. (a) An SEM image of cicada wing protrusions. (A) 3 dimensional, high resolution AFM image of the dorsal region of a cicada wing membrane (Pflatoda claripennis), and (B) corresponding height profile along a close-packed direction.

\subsection{Manipulative AFM and reflectance measurements}

By utilising manipulative atomic force microscopy, a confirmation of the function of the nano-array structures as an antireflective coating was possible. A high spring constant AFM probe was used to physically remove a section of the wing membrane. Figure 4 (A) and (B) show a 3 dimensional and topographical AFM image, respectively, of the resulting manipulation using a soft lever in order to avoid any further manipulation. The final result of manipulation comprised a volume of approximately $20 \times 20 \mu \mathrm{m}$ in the lateral dimensions and a depth of $300 \mathrm{~nm}$ being removed from the membrane surface. In this case the hard AFM probe was used as a 'nano-microtome' to physically remove membrane material. The optical image in figure 4 (C) clearly shows that the removal of the nano-structures produced a region on the membrane exhibiting higher reflective properties than the intact surrounding regions. As the process of membrane removal is a result of AFM probe/tip forces in the plane of the wing membrane as well as normal to the wing surface it is possible that the array structures are deformed/crushed during the manipulation process. SEM images of removed membrane regions indicate that the process depends on the AFM imaging conditions where sharp probes presumably were effective in controlling the nano-structure removal to produce varying heights. 

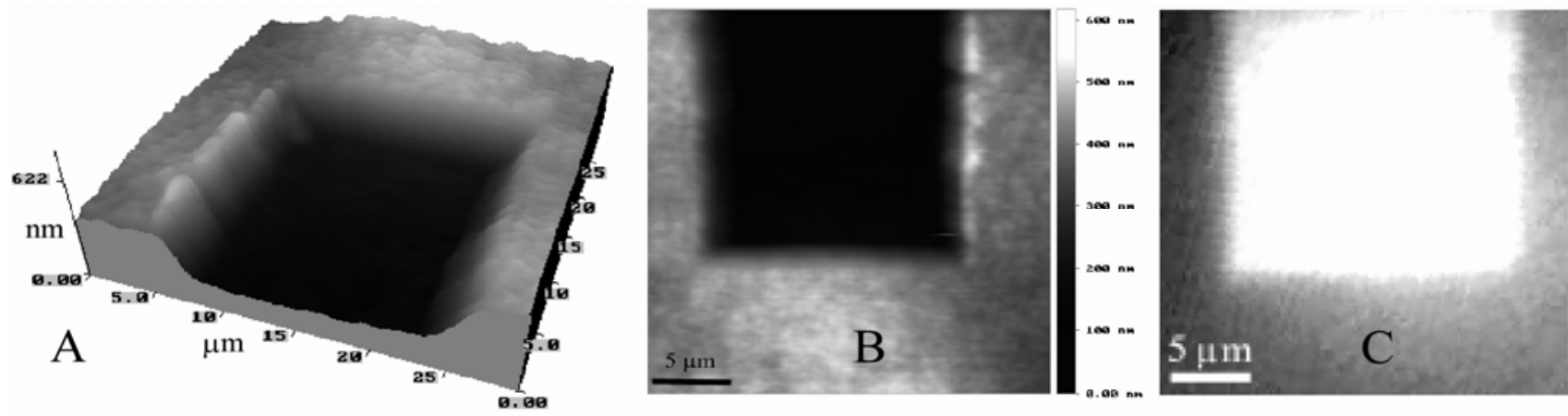

Figure 4 - (A) 3 dimensional image using a soft lever of a previously manipulated region using a stiff AFM lever on a cicada membrane. (B) shows the same region as in (A) grey-scale image. (C) Reflectance image of the manipulated section and surrounding intact region.

As briefly discussed in relation to the moth eye, by considering a periodic one dimensional profile it is possible to explain the optical properties of the subwavelength antireflective structures. A schematic representation of this scenario is presented in figure 5. If the period is larger than the radiation wavelength then the light is scattered into a number of waves, however if the grating is small when compared to the wavelength of incident light, only the zero-order diffracted wave propagates. Whether a diffraction order propagates or not is determined by the following grating equation ${ }^{7}$ :

$$
n \sin \theta_{m}-n_{i} \sin \theta_{i}=\frac{m \lambda}{\Lambda}
$$

where $n_{i}$ and $n$ are the indices of refraction for an incident medium and the medium where diffracted orders are propagating, respectively, where $n=n_{i}$ for reflected orders and $n=n_{s}$ for transmitted orders. $\theta_{i}$ and $\theta_{m}$ are the angle of incidence and the angle of the $m$ th order measured from the grating's surface normal, respectively. $\lambda=$ incident freespace wavelength, and $\Lambda=$ the period of the grating.

The ratio of grating period to wavelength can be obtained by manipulating equation 1 . If the $m=0$ order propagates in the incident medium or substrate, including all incident angles $\theta_{i}$ up to $\theta_{\max }$, at a given value of $\lambda$, the maximum angle of incidence yields the following expression giving an upper bound for the ratio:

$$
\frac{\Lambda}{\lambda}<\frac{1}{\max \left[n_{s}, n_{i}\right]+n_{i} \sin \theta_{\max }}
$$

where max $=$ the maximum of its arguments. An antireflection structured (ARS) surface period must always be less than the incident wavelength in order to avoid energy loss to diffracted orders, as stated by the inequality in equation 2 . For light with an incidence angle at 45 degrees onto a surface, with an $n$ value of 1.5 surrounded in an air medium, the largest period satisfying equation 2 would be around $200 \mathrm{~nm}$ for the visible spectrum. This is identical to the period observed for the nanostructures on the cicada wings.

According to thin film theory ${ }^{8}$ a single film coating will show zero reflectance at normal incidence provided its thickness is a quarter of a wavelength and its index of reflection satisfies the following:

$$
\begin{aligned}
& n_{\text {film }}=\left(n_{i} n_{s}\right)^{1 / 2} \\
& d=\frac{\lambda}{4\left(n_{i} n_{s}\right)^{1 / 2}}
\end{aligned}
$$

where $d$ is the structure height. Equation 3 gives us an approximation of the depth dimensions required for antireflective properties which are again smaller than the radiation wavelength of interest. 


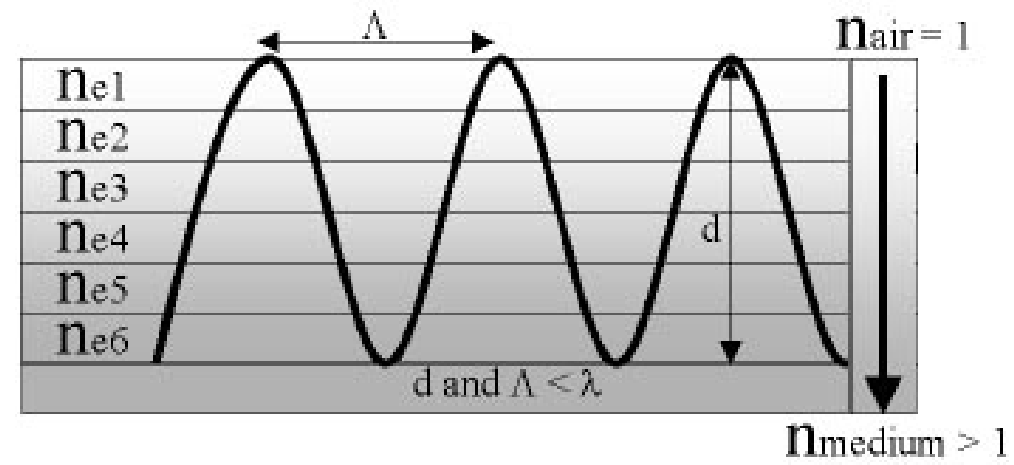

Figure 5. Schematic representation of a multi layered stack coating (gradient index coating) with each coating $\mathrm{n}_{\mathrm{e} 1}-\mathrm{n}_{\mathrm{e} 6}$ having an increasing refractive index. The periodic features represent a pseudo-gradient index coating comprising structures with a periodicity and height in the nanometre range where $\Lambda=$ the period between structures, $d=$ height of structures and $\lambda=$ wavelength of incident light.

By controlling the amount of membrane removed it was possible to determine the extent of reflectivity changes with depth. In essence the membrane was removed to produce a graduation in depth profiles of the nano-structures. Figure 6 shows an optical image with the five varying volumes of membrane material removed from the wing comprising depths of 200, 130, 300, 200 and $150 \mathrm{~nm}$ for squares A to E, respectively. Squares A and D (excavated regions of 200 and 300 $\mathrm{nm}$, respectively) show similar reflectivity intensities in the visible spectra. By removing $200 \mathrm{~nm}$ off the membrane surface, the resultant depth is of $25 \mathrm{~nm}$ (for a total depth of structures of $225 \mathrm{~nm}$ ) which offers very little reduction in reflections. At a depth of $130 \mathrm{~nm}$ (structure height $100 \mathrm{~nm}$ ) reflections are significantly reduced. The reflectance data clearly show the effect of structure height efficiency in acting as an anti-reflective coating. As the structures become smaller in height, the anisotropic gradient change in refractive index becomes less effective. Taller structures provide a more gradual change in the refractive index (from air, 1, to membrane 1.5 ) with the effect of reduced fresnel reflections. The inset shows the result of AFM-based lithography. A grid pattern was created on the wing membrane which also resulted in less efficient anti-reflective regions. This demonstrates the level of detail and resolution possible. It is also a demonstration of the degree of applications and flexibility of AFM-based micro/nano lithography.

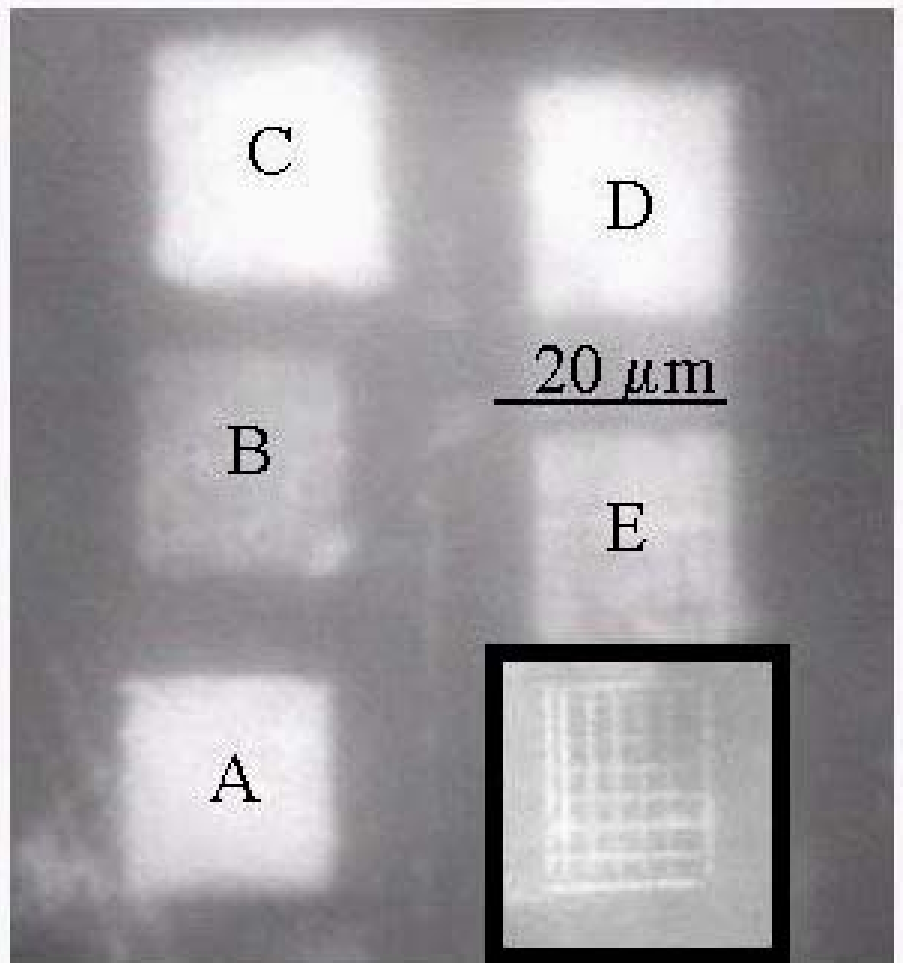

Figure 6 - (a) An optical image showing the extent of reflectivity as a result of AFM based manipulation using a high spring constant lever. Squares A to E represent manipulated membrane regions at depths of 200, 130, 300, 200 and 150 $\mathrm{nm}$, respectively. The highlighted square (bottom right) shows a grid pattern formed by AFM based lithography on the insect membrane. 


\subsection{Friction Analysis}

The intact and manipulated regions were also analysed in the lateral force mode in order to ascertain the adhesive and frictional properties. The topographical and frictional force images shown in figure 7 (a) and (b), respectively, were obtained using a soft lever imaged over of a manipulated section with a depth of $200 \mathrm{~nm}$ below the base of the nanostructures. This results in the total removal of the wing arrays. The frictional force image shows a much higher frictional force on the removed wing region. The quantitative friction loop data (friction force line profile) in figure 7 (c) show a frictional force of $30 \mathrm{nN}$ for the intact wing and $200 \mathrm{nN}$ for the removed section. The higher value indicates that the two regions may exhibit different surface chemistries. As the frictional force between the probe and the membrane is dependent on the probe/membrane contact conditions (e.g., contact geometry, sample compliance-indentation of probe into the surface membrane) the frictional difference may also be related to the change in nano-structure profile. Raman analysis of the membrane however showed that the intact regions showed more of a hydrocarbon (wax-like) nature than the excavated regions.

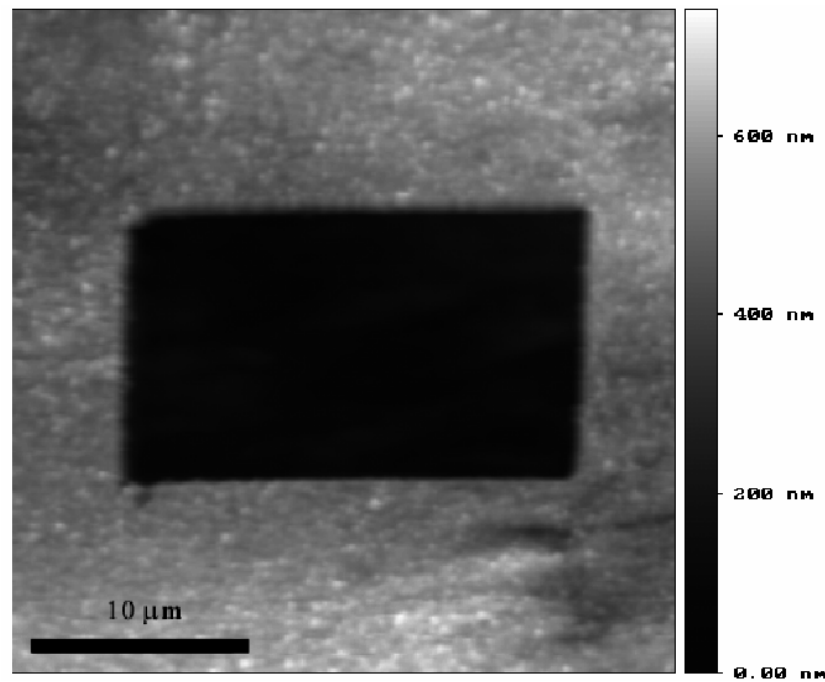

(a)

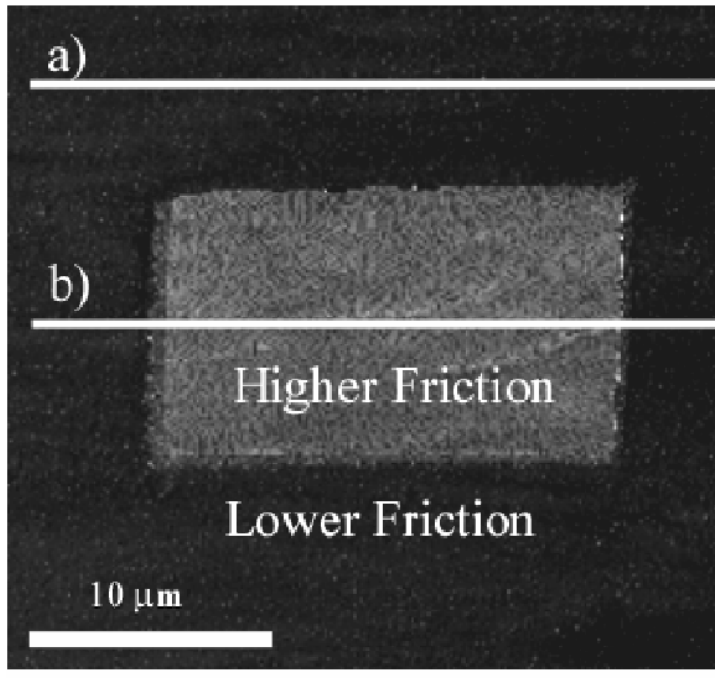

(b)

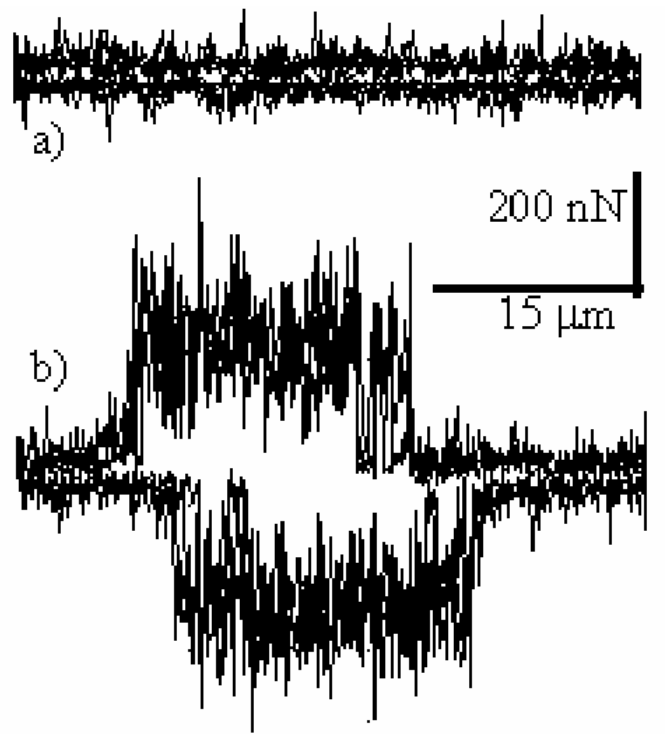

(c)

Figure 7 - (a) AFM grey scale topographical image of a previously AFM manipulated region on a cicada membrane. (b) Frictional force image of the same region as shown in (a). (c) Friction loop (friction profile) on corresponding two locations from the image (b). 
TEM images of the outer membrane often showed a thin layer beneath the membrane structures extending some 150nm showing similar electron density to the nano-structures. This is seen in figure 8 below. The wing was sectioned perpendicular to the plane of the wing and imaged edge on.

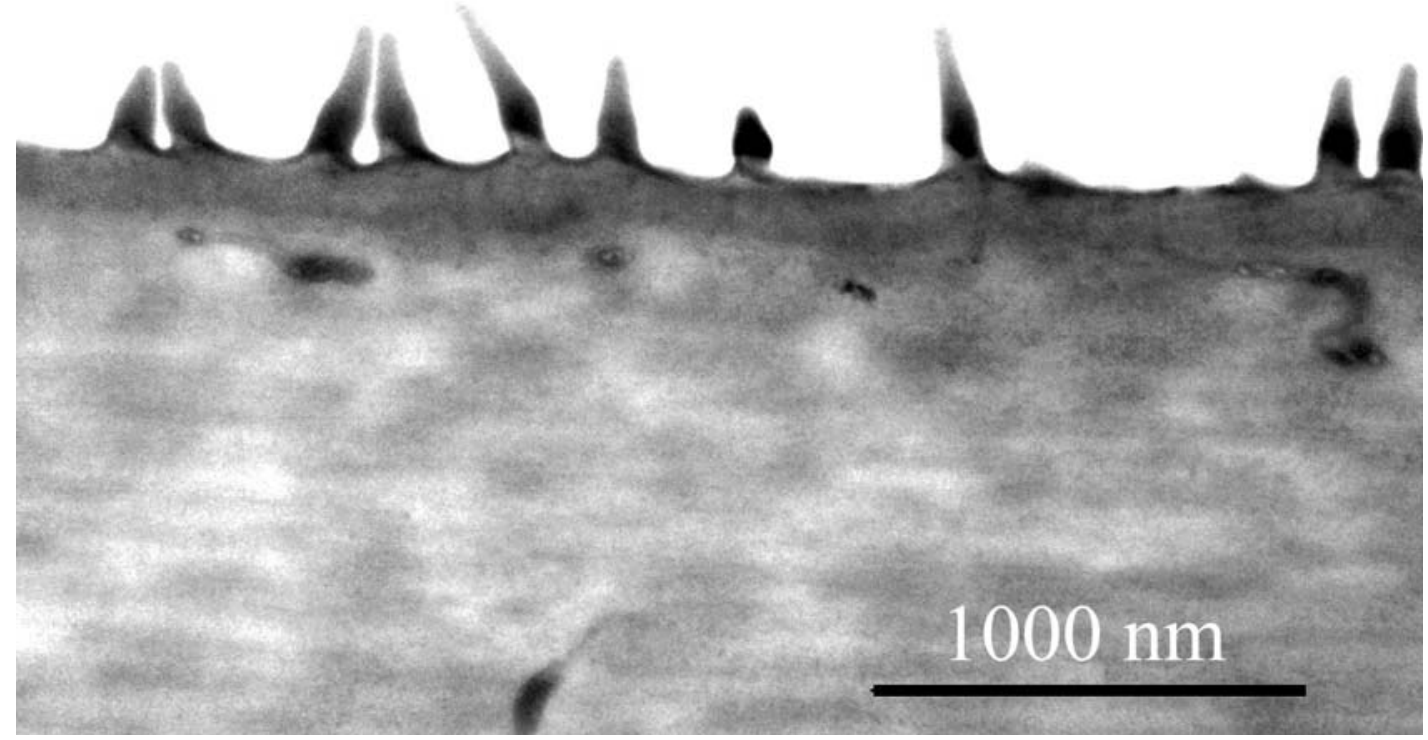

Figure 8 - TEM image of the outer membrane of the cicada wing.

The Raman and TEM data would indicate that the frictional contrast observed is a result of differing 'original' chemistry. Supporting data comes from AFM friction images where some of the nanostructure material was not completely during the manipulation process (total depth $520 \mathrm{~nm}$ ). Figure 9 shows frictional differentiation with two distinct frictional values. The partially removed material (which did not show the intact array shape at higher resolution) shows the same frictional values as the intact regions. F-d curve analysis of these debris regions also showed the same adhesive force as the intact regions.

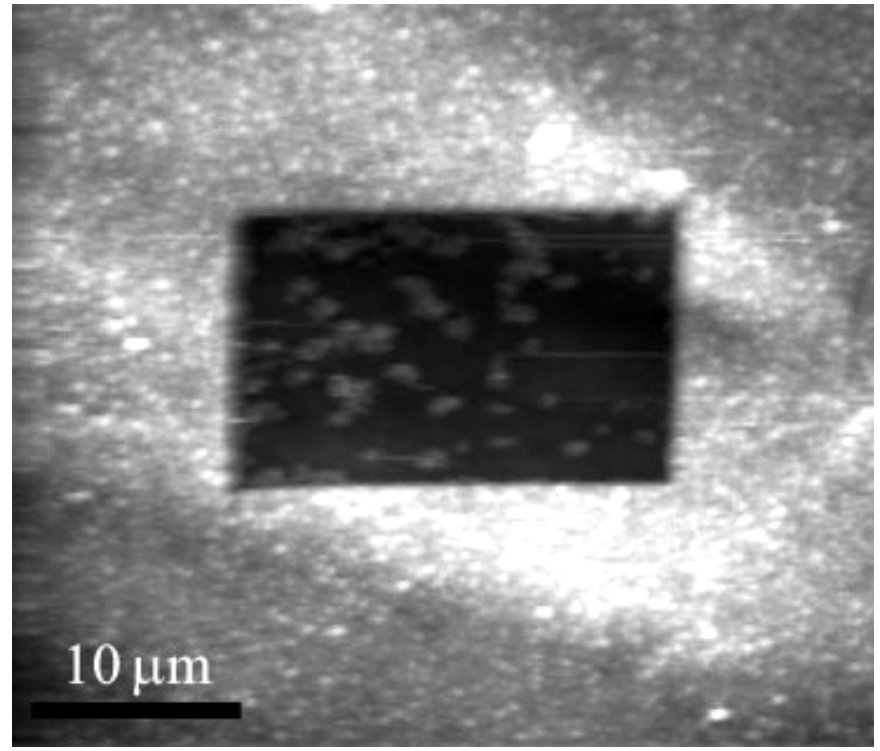

(a)

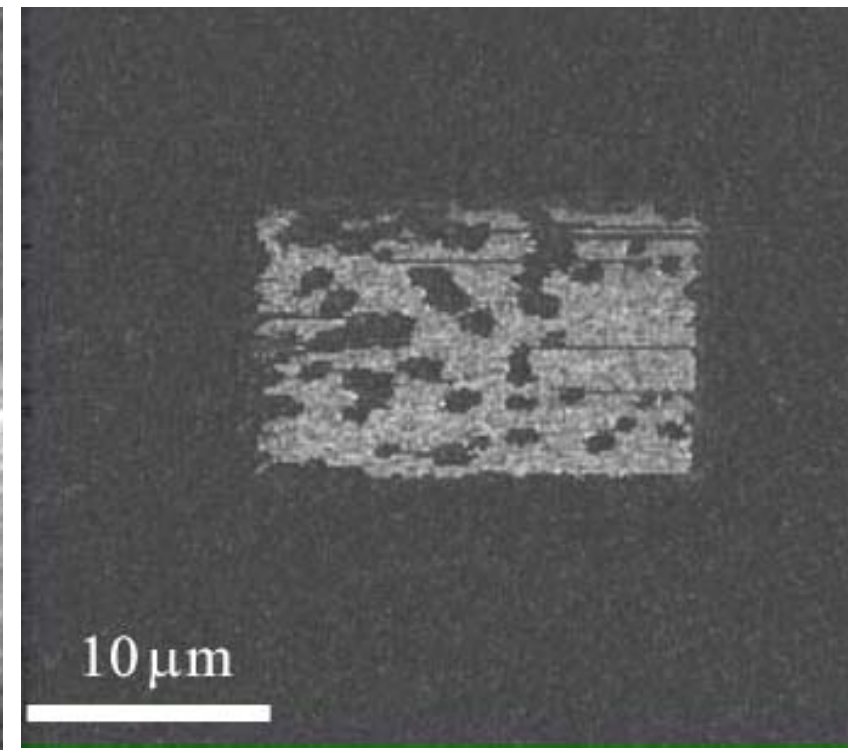

(b)

Figure 9. (a) AFM grey scale image of a previously AFM manipulated region on a cicada membrane. (b) Frictional force image of the same region as shown in (a). 


\section{CONCLUSION}

Evolution is quite ruthless in its choice of technologies. Those which succeed will confer a procreative advantage. On the other hand, many of those technologies serve similar purposes to those that confer advantages for the consuming enduser in our society such as the honeycomb structure which showed enormous impact in materials technology. Stealth is now the focus. The ability to control reflectance/transmittance by AFM manipulation of nano-structure arrays is an interesting outcome of this study. This technology could in principle be incorporated into man-made coatings (especially polymer array coatings) as a means of controlling optical properties on the micro-scale with precise tuning and spatial engineering. The anti-reflective coating is also tunable to some degree in terms of altering the exposed chemistry and also the geometry contact regime of the membrane surface. These parameters in turn will determine the reactivity of the surface.

\section{ACKNOWLEDGMENT}

The authors would like to thank Dr Bronwen Cribb for her assistance with the TEM and SEM data and helpful discussions.

\section{REFERENCES}

1. Bernhard CG, Miller WH, “A corneal nipple pattern in insect compound eyes”, Acta Physiologica Scandinavica, 56, 385-386, 1962.

2. Yoshida A, Motoyama M, Kosaku A, Miyamoto K, "Nanoprotuberance array in the transparent wing of a hawkmoth, Cephonodes hylas", Zoological Science [Short Communication], 13, 525-526, 1996.

3. Grann EB, Moharam MG, Pommet DA, "Optimal design for antireflective tapered two-dimensional subwavelength grating structures”, Journal of the optical society of America A, 12, 333-339, 1995.

4. Yu Z, Gao H, Wu W, Ge H, Chou SY, "Fabrication of large area subwavelength antireflection structures on Si using trilayer resist nanoimprint lithography and liftoff”, Journal of Vacuum Science and Technology B, 21, 2874-2877, 2003.

5. Bernhard CG, Miller WH, "Function of corneal nipples in compound eyes of insects", Acta Physiologica Scandinavica, 58, 381-382, 1963.

6. Bernhard CG, Miller WH, Møller AR, "The insect corneal nipple array. A biological, broad-band impedance transformer that acts as a antireflection coating”, Acta Physiologica Scandinavica, 63, 1-79, 1965.

7. Raguin DH, Morris GM, "Antireflection structured surfaces for the infrared spectral region”, Applied Optics, 32, 1154-1167, 1993.

8. Macleod HA, Thin-film optical filters, Chapters 1 \& 2, American Elsevier, New York, 1969. 